\title{
Clinico-Mycological Study of Dermatophytosis and Dermatomycosis in Tertiary Care Hospital
}

\author{
R. Tokbipi Phudang*, P. Baradkar Vasant and S. Shastri Jayanthi \\ Department of Microbiology, T.N.M.C. \& B.Y.L. Nair Ch. Hospital, Mumbai-400008, \\ Maharashtra, India \\ *Corresponding author
}

Keywords

Dermatophytosis, Dermatomycosis, Dermatophyte test medium

\section{Article Info}

Accepted:

10 December 2018 Available Online:

10 January 2019

A B S T R A C T

Aim of the study is to isolate and enumerate dermatophytes and other fungi from clinically suspected cases of Dermatomycoses, to co-relate the isolate and findings with clinical presentations and to analyse the Dermatophyte test medium for the growth of dermatophytes. Hundred (100) clinically suspected cases of dermatophytosis and dermatomycoses attending Dermatology O.P.D., was selected for the study including hair, skin and nail samples. It was a prospective and descriptive study. Direct microscopy was performed using KOH (10 and 20\%) and culture performed using Sabourauds dextrose agar (SDA), Dermatophyte test medium (DTM) and Corn meal agar. Data analysis was made on SPSS version 20, using chi-square test. The p value of 0.05 or less was considered significant. The highest age incidence was age group $21-30$ years. Tinea unguium (68\%) was the commonest clinical type followed by Tinea corporis $(13 \%)$. $\mathrm{KOH}$ was positive in $60(60 \%)$ cases and culture positivity in $45(45 \%)$ cases. Trichophyton mentagrophyte $(28.9 \%, 13 / 45)$ was the commonest dermatophyte isolated. Among the non-dermatophyte, Candida albicans $(17.8 \%, 8 / 45)$ was the commonest isolate, followed by Candida tropicalis $(15.6 \%, 7 / 45)$ and Candida parapsilosis $(13.3 \%, 6 / 45)$. DTM was not a good medium for primary isolation of dermatophytes in our study.

\section{Introduction}

Dermatophyte infection is a disease of worldwide distribution that accounts for majority of superficial infections. Lesion of skin includes tenia capitis, tinea cruris, tinea pedis, tinea barbae, tinea manuum, tinea faciei and tinea corporis. Hairs on scalp are more involved which may show two types of lesions i.e., ectothrix and endothrix. Nail of toes has maximum involvement. Dermatophytes possess the affinity for parasitizing keratin rich tissues like skin, hair and nails and produce dermal inflammatory response and intense itching in addition to a cosmetically poor appearance. The causative fungi colonize only cornified layer of epidermis or suprafollicular portions of hair and do not penetrate into deeper anatomical sites. The dermatophytes are among the commonest infectious agents of man. Dermatophytosis (tinea or ring worm), refers to infection of keratinised structures while dermatomycosis is infection caused by fungi, other than 
dermatophytes. Dermatophytosis is caused by three genera of dermatophytes, Microsporum, Trichophyton and Epidermophyton. The fungi which cause dermatomycosis are Candida spp., Aspergillus spp., Fusarium spp., Acremonium spp., Cladosporum, Scytalidium spp, etc. These isolates vary from place to place. Hot and humid climate in the tropical and subtropical countries like India makes dermatophytosis or ringworm a very common superficial fungal skin infection. It is common in tropics and may reach epidemic proportions in area with high rate of humidity, over population and poor hygienic conditions. Over the past decades, non-dermatophytes, as agents of superficial fungal infection in humans, produce lesions that are clinically similar to those caused by dermatophytic infections. Sabourauds dextrose agar is used for primary isolation of fungi. The dermatophyte test medium (DTM) is an alternative culture method that can be used to confirm a diagnosis of dermatophytosis. The culture medium was originally described by Taplin et al., Isolation of causative agent is important as the therapy is based upon the isolates. Hence, the knowledge of the causative agents from our locality is important for institution of appropriate therapy. Considering this our study has been planned.

\section{Materials and Methods}

A total of 100 clinically suspected cases of dermatophytosis and dermatomycoses attending the outpatient department of Dermatology in tertiary care hospital was selected for the study including samples of hair, skin and nail. The study was carried out for one and a half year duration from July 2014 to Dec 2015. The study and data accumulation were carried out with approval from Ethics Committee for Academic Research projects (ECARP) and informed consent was taken from the subjects. The age group of 18 years and above was included in the study. Suspected lesion was cleaned with $70 \%$ alcohol and allowed to dry. Using the blunt side of sterile scalpel, the skin and scalp scraping was collected from the active margin of the lesion. In addition a few affected hairs were also epilated and collected with a pair of sterilized tweezers. Care was taken to collect the basal portion of the hair as the fungus was usually found in this area. The affected area was cleansed with $70 \%$ ethyl alcohol and the nail specimen was collected by taking clippings of the infected part and scrapings beneath the nail. The specimens were collected on a sterile petri dish and transported within half an hour to Microbiology Department. The nail samples were placed in few drops of freshly prepared $20 \% \mathrm{KOH}$ in a test tube and kept at room temperature for overnight and observed under the microscope next day. Skin and hair samples were placed in a drop of $10 \% \mathrm{KOH}$, covered with coverslip and left at room temperature for 30 mins and observed under low power followed by high power microscope to see the presence of fungal element, septate and branching. The specimens were then inoculated on one set of Sabourauds dextrose agar with and without cycloheximide. One of the set was incubated at room temperature and the other was incubated at $37^{\circ} \mathrm{C}$ in the incubator. Part of each specimen was inoculated on Dermatophyte test medium. The culture (Sabourauds dextrose agar) were observed for growth daily in first week then twice weekly till 6 weeks. The colony characteristics like colour, texture, surface, shape and presence of pigment on reverse side of the slant were noted. Similarly Dermatophyte test medium was observed for colour change and if colour change occurs, the fungus was identified by lactophenol cotton blue preparation and slide culture on potato dextrose agar. This procedure is also followed for any fungal growth on Sabourauds dextrose agar slants. The cultures were examined microscopically by removing a portion of the aerial mycelium 
with a sterile straight wire, placed on a glass slide in a drop of Lactophenol cotton blue and a coverslip is placed by avoiding air bubbles. The wet mount was observed under low power and high power of the microscope and different morphologic types of fungi were identified depending on the hyphae hyaline or dematiaceous, septate or non-septate, morphology and arrangement of macroconidia and microconidia. Urease test using Christensen's urea agar was performed whenever necessary. The yeast isolate were identified by Germ tube test, growth pattern on corn meal agar (Dalmau method) and Sugar assimilation test using glucose, lactose, sucrose, maltose, cellobiose and dulcitol. Statistical analysis was done on SPSS version 20 , using chi-square test. The $\mathrm{p}$ value of 0.05 or less was considered significant.

\section{Results and Discussion}

The age group of patients in the study ranged from $18-88$ years. The most common age group was $21-30$ years, followed by $31-40$ years and $41-50$ years with male to female ratio 1:1. Among the 100 cases, 26 were skin scrapings, 68 were nail clippings and 6 were hair samples.

A comparison of the direct microscopy and culture results is shown in Table 1. Out of 100 samples examined, fungal elements were seen on direct microscopy in 60(60\%) cases and culture was positive in $45(45 \%)$ cases. Forty (40\%) cases were both $\mathrm{KOH}$ and culture positive. Twenty (20\%) cases were $\mathrm{KOH}$ positive but culture negative whereas, $5(5 \%)$ cases were $\mathrm{KOH}$ negative but culture positive. Thirty-five (35\%) cases were both $\mathrm{KOH}$ and culture negative. Chi square test was applied which was statistically significant ( $\mathrm{p}$ value $<0.05)$.

Clinical types of dermatophytosis and dermatomycosis in different age group are given in Table 2. The results of fungal culture in different clinical types are given in Table 3 . Of the 6 hair samples, only $1(16.67 \%, 1 / 6)$ grew Trichosporon species. Among 26 skin samples, $12(46.15 \%, 12 / 26)$ were culture positive. Of these, $9(34.6 \%, 9 / 26)$ were dermatophytes and 3(11.5\%, 3/26) were nondermatophyte. Among 68 nail samples, $32(47.1 \%, 32 / 68)$ were culture positive. Of these, $6(8.8 \%, 6 / 68)$ were dermatophytes and $26(38.2 \%$, 26/68) were non-dermatophytes. Among the isolates, Trichophyton mentagrophyte $(28.9 \%, 13 / 45)$ was the commonest dermatophyte isolated. Among non-dermatophyte, Candida albicans $(17.8 \%$, $8 / 45)$ was the commonest, followed by Candida tropicalis $(15.6 \%, 7 / 45)$ and Candida parapsilosis $(13.3 \%, 6 / 45)$.

Of 68 Tinea unguium (32 culture positive, 42 $\mathrm{KOH}$ positive), Candida albicans $(8,11.8 \%$, $8 / 68$ ) was the commonest isolate, followed by Candida tropicalis (7, 10.3\%, 7/68), and Trichophyton mentagrophyte $(5,7.4 \%, 5 / 68)$ and Candida parapsilosis $(5,7.4 \%, 5 / 68)$. Of 13 Tinea corporis ( 7 culture positive, $10 \mathrm{KOH}$ positive), Trichophyton mentagrophyte (6, $46.2 \%, 6 / 13$ ) was the commonest isolate (Fig. 1 and 2).

Hundred (100) clinically suspected cases of dermatophytosis and dermatomycosis attending Dermatology OPD from July 2014 to Dec 2015 were included in the study. Age group of 18 and above and both sexes were included. In our study the most common age group among the 100 analysed were 21-30 years $(36 \%)$ followed by $31-40$ years $(20 \%)$ and 41-50 years (17\%). Nilekar et al., and Vignesh et al., reported similar observations. Post pubertal changes in hormones resulting in acidic sebaceous gland secretions are responsible for decrease in incidence with age. Sharma et al., reported that the commonest age group in their study was 31-40 years $(31.25 \%)$. In our study, the male to female 
ratio was 1: 1, a finding similar to Araj et al., But most of the studies like Sharma et al.,; Nilekar et al., and Vignesh et al., show male preponderance. Most of the studies show male preponderance but in our study males and females were affected equally. This may be due to increased participation of women in outdoor activities, use of footwear and higher degree of health awareness.

The commonest clinical type in the present study was Tinea unguium 68\% (68/100) and was found highest in age group 21-30 years $(33.8 \%, 23 / 68)$. These could be due to trauma inflicted to nails as a result of hard physical work and habit of walking and working barefooted. In our study, Tinea unguium $(68 \%, 68 / 100)$ was followed by Tinea corporis $(13 \%, 13 / 100)$. The pruritic nature of Tinea corporis, lead to seeking of medical attention by the patients. Tinea unguium was also found highest in Ghosh et al., (74.58\%) while studies like Nilekar et al., (32.5\%); Santosh et al., (32.21\%) and Bindu et al., (54.6\%) has Tinea corporis as the major clinical type.

In the present study, direct microscopy by $\mathrm{KOH}$ was positive in $60(60 \%)$. Bindu et al., also reported similar $\mathrm{KOH}$ positivity of $64 \%$. Mistry et al., (86.86\%) and Ghosh et al., $(91.34 \%)$ reported with higher $\mathrm{KOH}$ positivity as compared to our study. While Santosh et al., reported with low $\mathrm{KOH}$ positivity of
$55.37 \%$ as compared to our study. This may be due to non-viability of the fungi due to application of antifungal agent prior to sample collection or could be due to absence of fungal element in the portion of sample used for culture. Culture positivity in our study was 45 $(45 \%)$. Similar culture positivity was seen in studies like Bindu et al., (45.3\%) Error! Reference source not found.; Santosh et al., (46.97\%); Nilekar et al., (45.62\%). Other studies that showed higher culture positivity rate were Ghosh et al., (87.43\%); Omar et al., (55\%); Chudasama et al., (59.5\%). In our study $20(20 \%)$ cases were $\mathrm{KOH}$ positive but culture negative. Such observation was also seen by Mahale et al., and Dodamani et al., While $5(5 \%)$ cases in our study were $\mathrm{KOH}$ negative but culture positive, this could be due to the inactive sporulating phase of fungi that is difficult to be viewed under microscope, an observation done by Mahale et al., too. Forty (40\%) cases were both $\mathrm{KOH}$ and culture positive and $35 \%$ were both $\mathrm{KOH}$ and culture negative in the present study. Chi square test was applied which was statistically significant ( $\mathrm{p}$ value $<0.05$ ) (Table 1). In the present study, $20(20 \%)$ specimens were positive for $\mathrm{KOH}$ alone and $5(5 \%)$ were positive by culture alone, highlighting the importance of both microscopy and culture in the definitive diagnosis of dermatophytosis and dermatomycosis (Fig. 3-9).

Table.1 Results obtained after direct examination and culture

\begin{tabular}{|l|l|l|l|}
\hline & $\begin{array}{l}\text { KOH POSITIVE } \mathbf{n} \\
(\boldsymbol{\%})\end{array}$ & $\begin{array}{l}\text { KOH NEGATIVE } \mathbf{n} \\
(\boldsymbol{\%})\end{array}$ & TOTAL \\
\hline $\begin{array}{l}\text { CULTURE } \\
\text { POSITIVE }\end{array}$ & $40(40)$ & $5(5)$ & 45 \\
\hline $\begin{array}{l}\text { CULTURE } \\
\text { NEGATIVE }\end{array}$ & $20(20)$ & $35(35)$ & 55 \\
\hline TOTAL & $60(60)$ & $40(40)$ & 100 \\
\hline
\end{tabular}


Table. 2 Clinical types of dermatophytosis and dermatomycosis with reference to clinical manifestation (Type) in different age group (Years)

\begin{tabular}{|l|l|l|l|l|l|l|l|}
\hline \multicolumn{1}{|c|}{ Clinical types } & $\begin{array}{l}\text { Patient } \\
\text { samples }\end{array}$ & $\begin{array}{l}\mathbf{1 8 - 2 0} \\
\mathbf{n}(\boldsymbol{\%})\end{array}$ & $\begin{array}{l}\mathbf{2 1 - 3 0} \\
\mathbf{n}(\boldsymbol{\%})\end{array}$ & $\begin{array}{l}\mathbf{3 1 - 4 0} \\
\mathbf{n}(\%)\end{array}$ & $\begin{array}{l}\mathbf{4 1 - 5 0} \\
\mathbf{n}(\%)\end{array}$ & $\begin{array}{l}\mathbf{5 1 - 6 0} \\
\mathbf{n}(\%)\end{array}$ & $\begin{array}{c}>\mathbf{6 0} \\
\mathbf{n}(\%)\end{array}$ \\
\hline Tinea unguium & $68(68)$ & $2(2.9)$ & $23(33.8)$ & $15(22.1)$ & $13(19.1)$ & $3(4.4)$ & $12(17.6)$ \\
\hline Tinea corporis & $13(13)$ & $3(23.1)$ & $4(30.8)$ & $4(30.8)$ & $1(7.7)$ & $1(7.7)$ & - \\
\hline Tinea capitis & $6(6)$ & $2(33.33)$ & $3(50)$ & - & $1(16.7)$ & - & - \\
\hline Tinea cruris & $4(4)$ & - & $3(75)$ & - & $1(25)$ & - & - \\
\hline Tinea pedis & $4(4)$ & $1(25)$ & - & - & $1(25)$ & $1(25)$ & $1(25)$ \\
\hline Tinea manuum & $2(2)$ & - & $1(50)$ & - & - & $1(50)$ & - \\
\hline Tinea faciei & $1(1)$ & - & $1(100)$ & - & - & - & - \\
\hline Tinea barbae & $1(1)$ & - & - & $1(100)$ & - & - & - \\
\hline $\begin{array}{l}\text { Tinea cruris }+ \\
\text { Tinea corporis }\end{array}$ & $1(1)$ & - & $1(100)$ & - & - & - & - \\
\hline Total & 100 & 8 & 36 & 20 & 17 & 6 & 13 \\
\hline
\end{tabular}

Table.3 Identification of dermatophytosis and dermatomycosis by microscopy and culture among clinical types

\begin{tabular}{|c|c|c|c|c|c|c|c|c|c|c|c|c|}
\hline 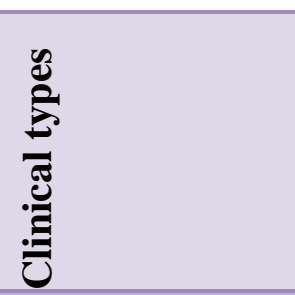 & 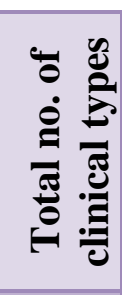 & 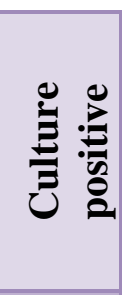 & 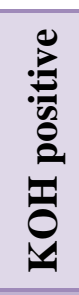 & 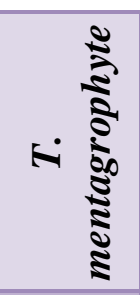 & 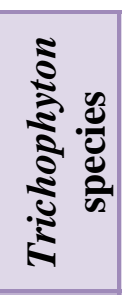 & 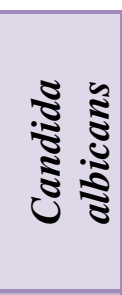 & 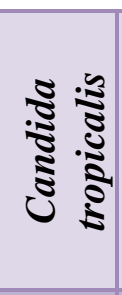 & 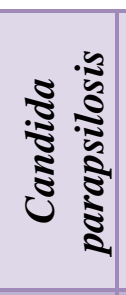 & 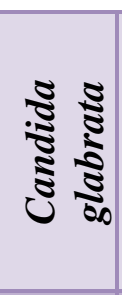 & 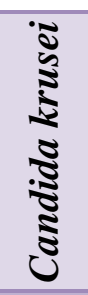 & 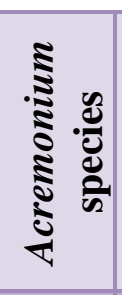 & 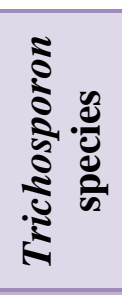 \\
\hline Tinea unguium & 68 & 32 & 42 & 5 & 1 & 8 & 7 & 5 & 3 & 1 & 1 & 1 \\
\hline Tinea corporis & 13 & 7 & 10 & 6 & - & - & - & - & - & - & 1 & - \\
\hline Tinea capitis & 6 & 1 & 2 & - & - & - & - & - & - & - & - & 1 \\
\hline Tinea cruris & 4 & 1 & 1 & - & 1 & - & - & - & - & - & - & - \\
\hline Tinea pedis & 4 & 1 & 2 & - & - & - & - & 1 & - & - & - & - \\
\hline Tinea manuum & 2 & 1 & - & - & - & - & - & - & - & - & 1 & - \\
\hline Tinea faciei & 1 & 1 & 1 & 1 & - & - & - & - & - & - & - & - \\
\hline Tinea barbae & 1 & - & 1 & - & - & - & - & - & - & - & - & - \\
\hline \multirow[t]{2}{*}{$\begin{array}{l}\text { Tinea cruris + } \\
\text { Tinea corporis }\end{array}$} & 1 & 1 & 1 & 1 & - & - & - & - & - & - & - & - \\
\hline & 100 & 45 & 60 & 13 & 2 & 8 & 7 & 6 & 3 & 1 & 3 & 2 \\
\hline
\end{tabular}


Fig.1 Tinea unguium (destruction of nail plates) and Tinea corporis (annular scaly plaques with advancing margins)
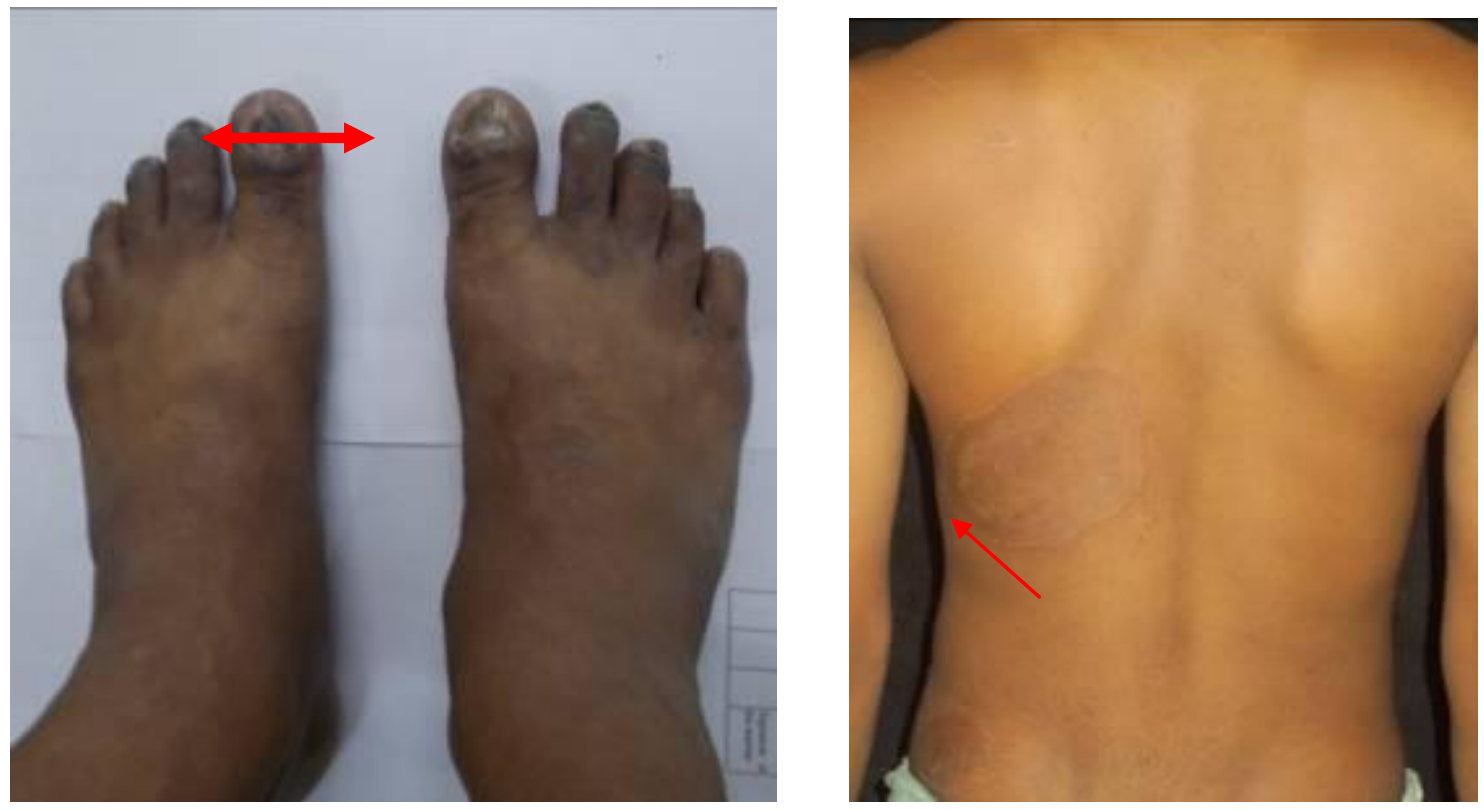

Fig.3\&4 KOH mount showing hyaline septate hyphae and arthroconidia (X400) and LPCB mount of Trichophyton mentagrophyte showing spiral hyphae (X 400)
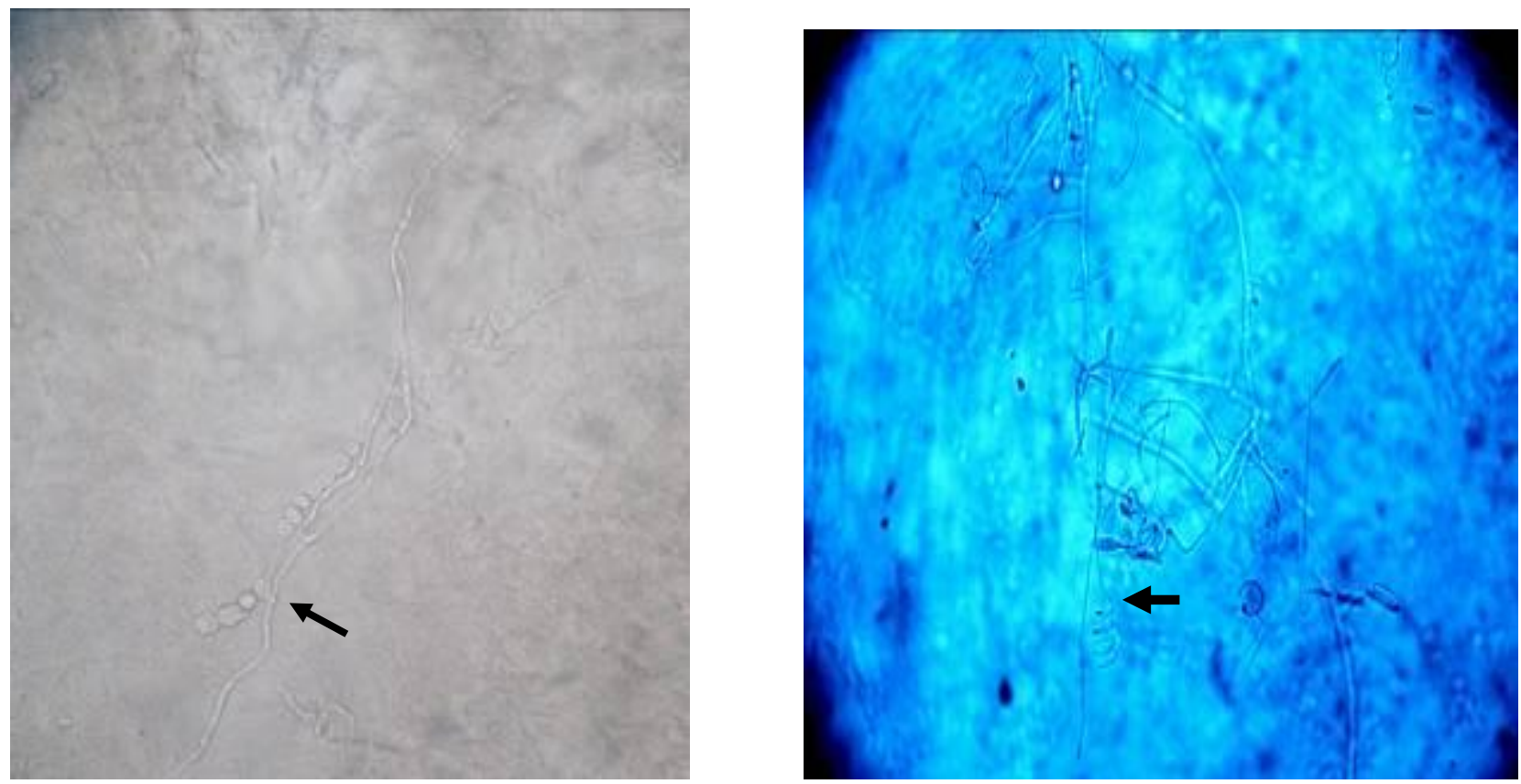
Fig.5 SDA with growth of Trichophyton mentagrophyte showing white powdery colony on obverse side and brownish tan pigment on reverse
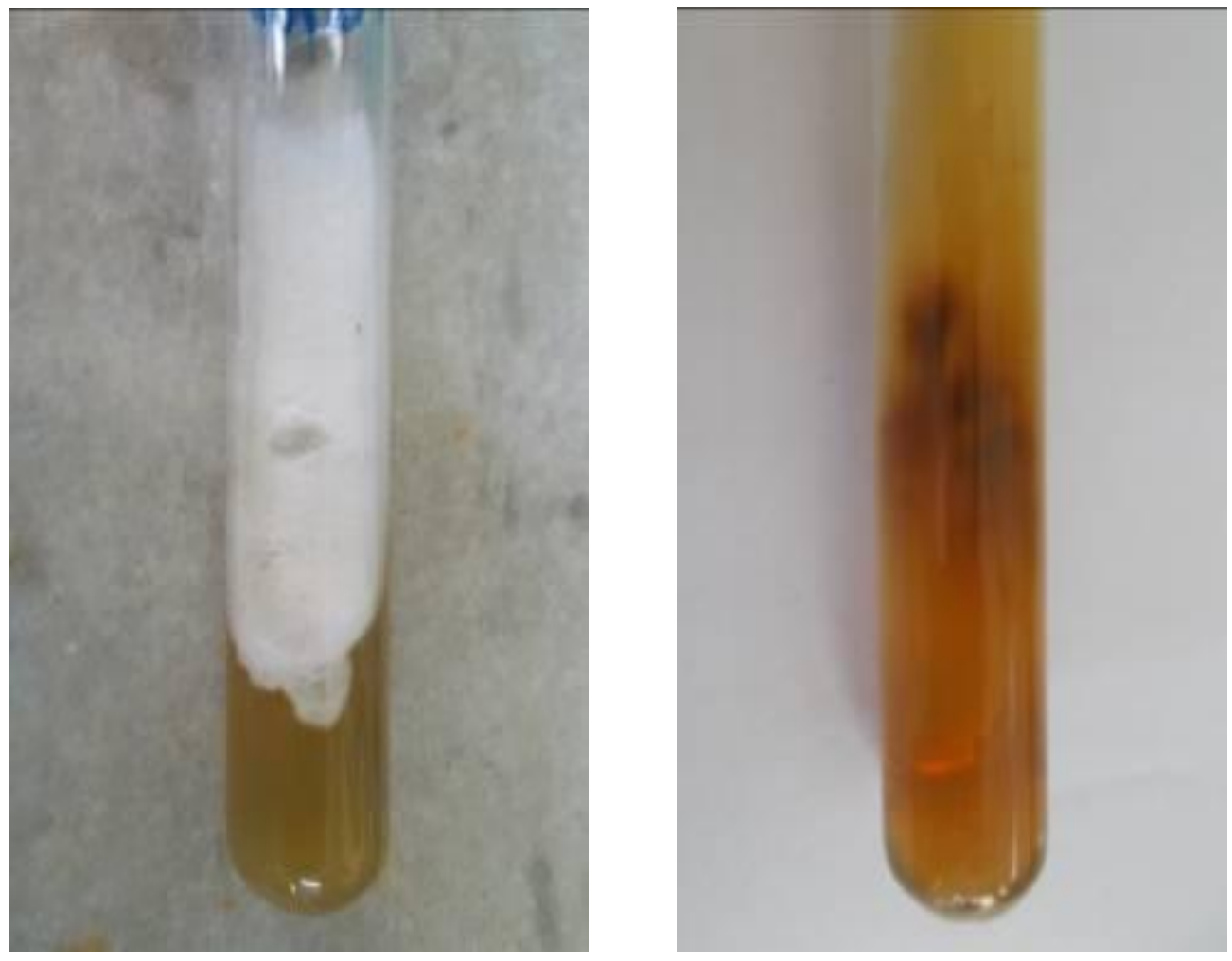

Fig.6\&7 SDA showing colony of Candida albicans and Germ tube test (x400)
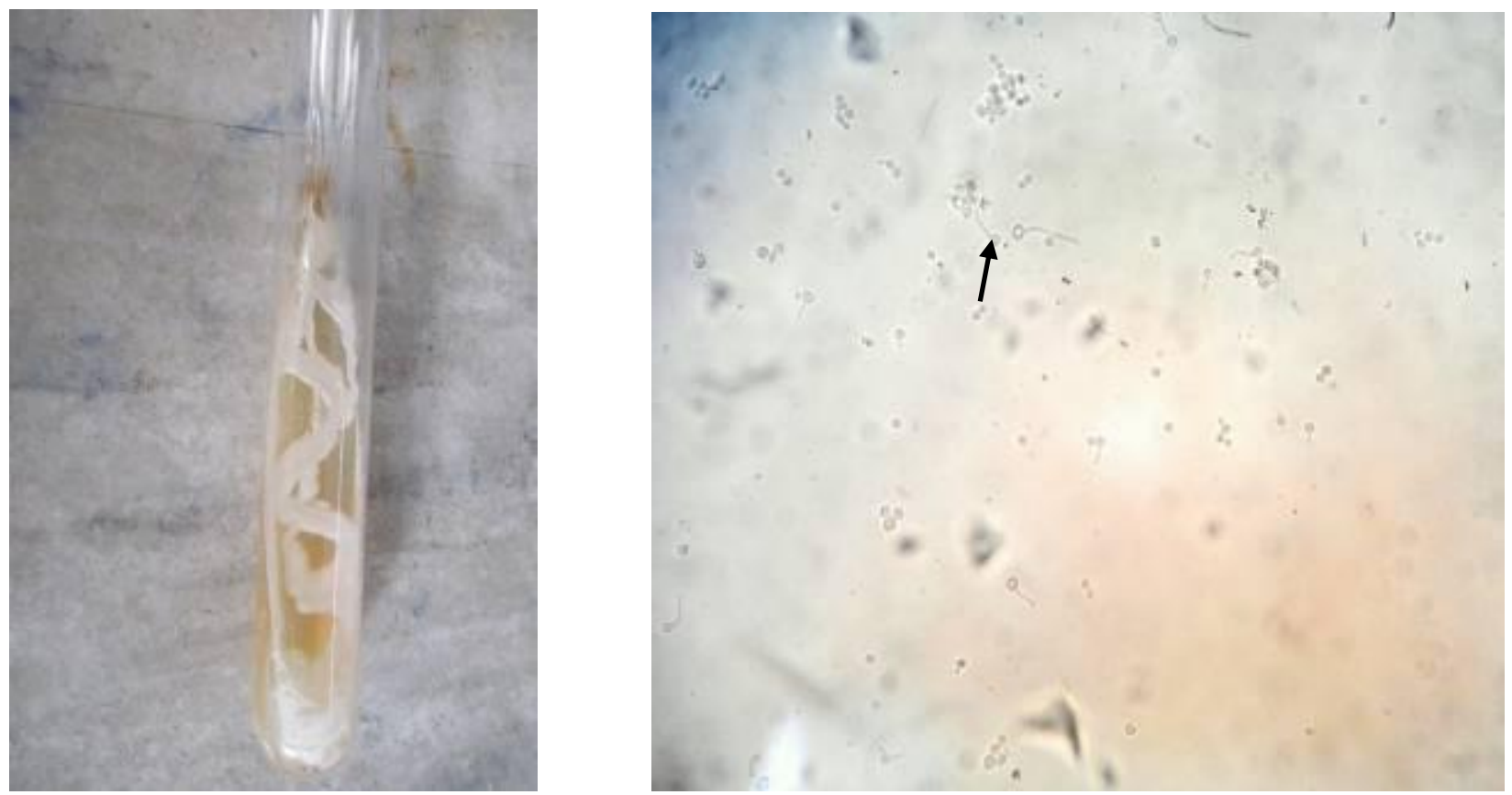
Fig.8 Dalmau culture on cornmeal agar showing pseudohyphae, clusters of blastoconidia and terminal chlamydospores of Candida albicans (x400).

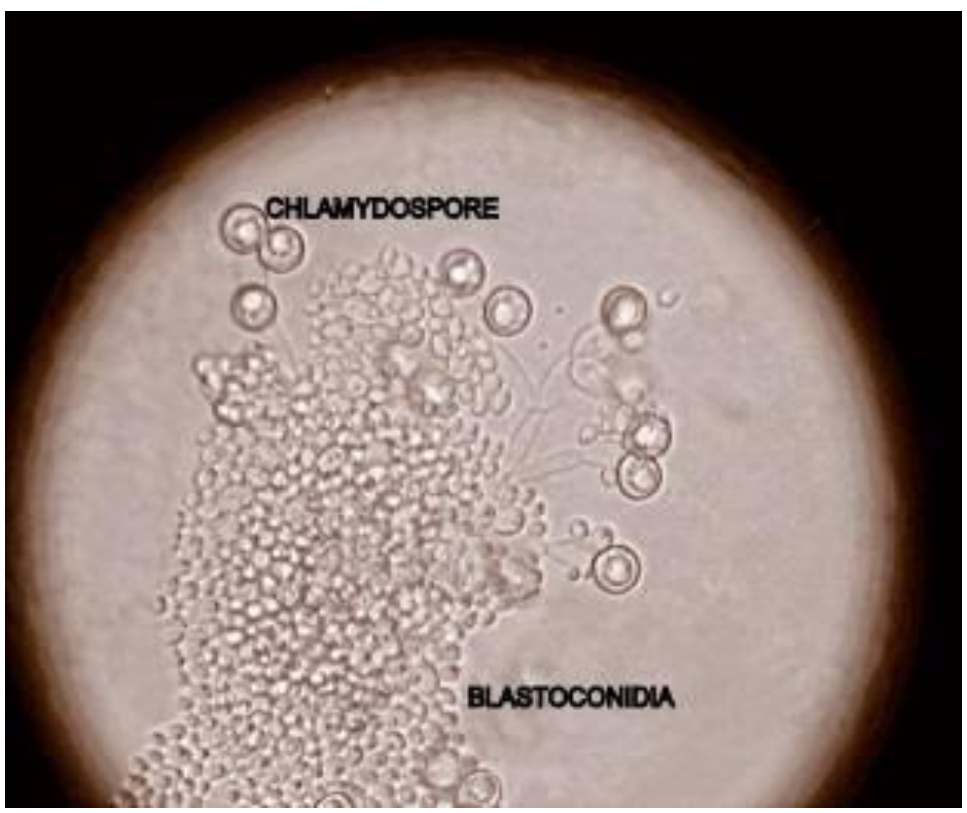

Fig.9 Sugar assimilation test (Candida tropicalis) (growth around cellobiose, glucose, sucrose and maltose are seen as white opacity)

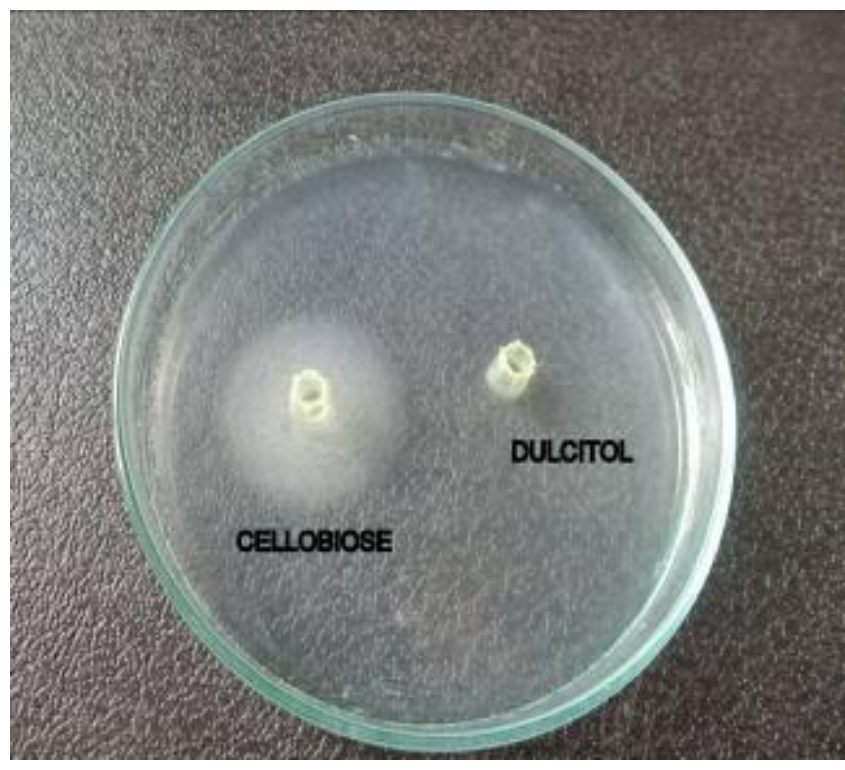

In the present study, Tinea unguium was the most common clinical type. The isolates from tinea unguium were Candida albicans (17.8\%, 8/45), Candida tropicalis (15.6\%, 7/45), Trichophyton mentagrophyte (11.1\%, 5/45) and Candida parapsilosis (11.1\%,

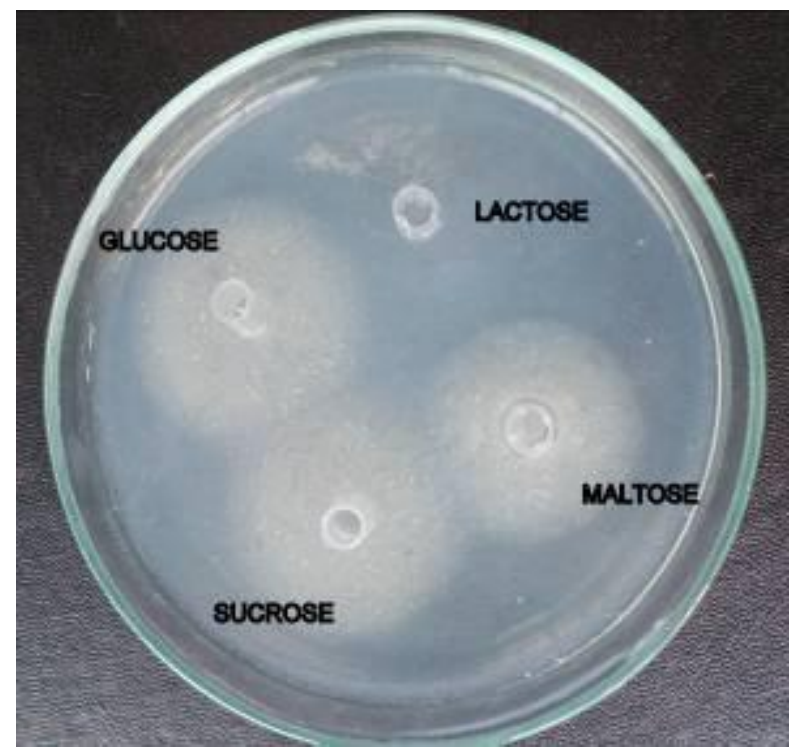

5/45). The isolates in the study done by Nasimuddin et al., from tinea unguium were Trichophyton mentagrophyte $(39.4 \%, 13 / 33)$, Trichophyton rubrum $(33.3 \%, 11 / 33)$ and Trichophyton tonsurans $(9.1 \%, 3 / 33)$. The isolate from tinea unguium reported by Mistry 
et al., were Trichophyton rubrum (54.5\%, 6/11) and Trichophyton mentagrophyte (36.4\%, 4/11). In our study, Tinea corporis was the second most common clinical type. The isolates from tinea corporis were in the following order: Trichophyton mentagrophyte $(13.33 \%, 6 / 45)$ and Acremonium species $(2.2 \%, 1 / 45)$. Nasimuddin et al., reported the following order from tinea corporis: Trichophyton rubrum (54.34\%), Trichophyton mentagrophyte (28.26\%) and Trichophyton tonsurans $(5.98 \%)$.

In the present study, growth of $15 \%$ dermatophyte and $30 \%$ non-dermatophyte were seen. Among the isolates, Trichophyton mentagrophyte $(28.9 \%, 13 / 45)$ was the commonest dermatophyte isolated. Trichophyton mentagrophyte was also found to be the commonest isolate in a study done by Nasimuddin et al., (38.75\%). While most of the studies shows Trichophyton rubrum to be the commonest isolate followed by Trichophyton mentagrophyte. This could be due to increased migration and climatic conditions as reported earlier. ${ }^{\text {Error! Bookmark not }}$ defined.

Among the non-dermatophyte, Candida albicans was the commonest isolate in the present study, followed by $C$. tropicalis and C. parapsilosis. In our study, among nondermatophyte Candida glabrata (10\%, 3/30), Candida krusei $(3.3 \%, 1 / 30)$, Trichosporon species $(6.7 \%, 2 / 30)$ and Acremonium species $(10 \%, 3 / 30)$ were also grown. The isolate was confirmed as pathogen by repeated isolation.

In the present study, out of one hundred specimens, 33\% (15/45) of dermatophytes were grown on Sabourauds dextrose agar while there was no growth of dermatophyte on Dermatophyte test medium ( $\mathrm{p}$ value < 0.05). Growth of organisms like Aspergillus species, Penicillium species etc. were seen, with change of colour of the medium from yellow to red due to change in $\mathrm{pH}$ of the media. These organisms were isolated from samples which were $\mathrm{KOH}$ negative and no growth on Sabourauds dextrose agar. Simultaneously, after subculture of the isolated dermatophyte from Sabourauds dextrose agar on Dermatophyte test medium, there was growth with the change in the colour of the media to red. Reporting of Dermatophyte test medium for primary isolation varied from one study to another. Walke et al., reported that Sabouraud Dextrose Agar showed growth of dermatophytes in $53.05 \%$ cases while Dermatophyte Test Medium isolated 54.34\% cases. False positive results were obtained in dermatophyte test medium due to colour change produced by growth of nondermatophytes such as Aspergillus spp., Penicillium spp. which was similar to study done by Salkin et al., and Sumathi et al., Rosenthal et al., reported that the commercial DTMs cannot be recommended as utterly satisfactory routine isolation media, where good laboratory facilities are available, but may be useful under less favourable clinical or field conditions. The clinical presentation though typical of ringworm infection is very often confused with other skin disorders particularly due to topical application of steroid ointments, leading to further misdiagnosis and mismanagement. Hence there arises the need for correct, efficient and rapid laboratory diagnosis of dermatophytes and also for the increasing non-dermatophyte infection.

\section{References}

1. Bindu V, Pavithran K. Clinico - mycological study of dermatophytosis in Calicut. IJDVL 2002; 68: 259-261.

2. Chudasama V, Solanki H, Vadsmiya M, Javadekar T. A Study of Superficial Mycosis in Tertiary Care Hospital. IJSR 2014; 3(3): 222-224.

3. Nilekar SL, Kulkarni VL. Dermatophytosis 
in and around Ambojogai. IOSR-JDMS 2015; 14(10):37-41.

4. Vignesh D, Sankar SP, Sudha S. A clinical study of a superficial dermatophytic infection in Kanchipuram. National Journal of Medical Research \& Yoga Science 2015; 1(2): 22-24.

5. Sharma Y, Jain S, Chandra K, Khurana VK, Kudesia M. Clinico-mycological evaluation of dermatophytes and non-dermatophytes isolated from various clinical samples: A study from north India. J Res Med Sci. 2012; 17(8): 817-818.

6. Araj GF, Racoubian ES, Daher NK. Etiologic agents of dermatophyte infection in Lebanon. J Med Liban 2004;52 (2): 59-63.

7. Santosh HK, Kandati J, Rao AVM, Buchineni M, Pathapati RM. Clinicomycological study of dermatophytosis - our experience. IJCMAS 2015; 4(7): 695-702.

8. Ghosh RR, Ray R, Ghosh TK, Ghosh AP. Clinico-mycological profile of Dermatophytosis in tertiary care hospital in West Bengal- an Indian scenario. IJCMAS 2014; 3(9): 655-666.

9. Mistry MA, Goswami YS, Rathod B, Dalwadi PH. Clinico-mycological studies of dermatophytosis at tertiary care centre, West India. Int J Bio Med Res 2014; 5(4): 45564561.

10. Omar BJ, Agnihotri P, Upadhyay GC, Sakhuja S, Arora SK. Non dermatophytic fungal infections amongst the dermatophytosis- A hospital based study. IJCH 2013; 25 (1): 34-38.

11. Mahale RP, Rao MR, Tejashree A, Deepashree R, Kulkarni M.
Clinicomycological profile of dermatophytosis in a teaching hospital. IJPSI 2014; 3(8): 43-46.

12. Doddamani PV, Harshan KH, Kanta RC, Gangane R, Sunil KB. Isolation, identification and prevalence of dermatophytes in tertiary care Hospital in Gulbarga District. PJSR 2013; 6(2): 10-13.

13. Nasimuddin S, Appalaraju B, Surendran P, Srinivas CR. Isolation, identification and comparative analysis of SDA and DTM for dermatophytes from clinical samples in a tertiary care hospital. J Den Med Sci 2014; 13(11): 68-73.

14. Kannan P, Janaki C, Selvi GS. Prevalence of dermatophytes and other fungal agents isolated from clinical samples. IJMM 2006; 24(3): 212-5.

15. Walke HR, Gaikwad AA and Palekar SS. Clinico-mycological profile of dermatophytosis in patients attending dermatology OPD in tertiary care hospital, India. Int J Curr Microbiol App Sci., 2014; 3(10): 432-440.

16. Salkin I F. Dermatophyte Test Medium: Evaluation with Nondermatophytic Pathogens. Applied microbiology 1973; 26 (2): 134-137.

17. Sumathi S, Mariraj J, Shafiyabi S, Ramesh R, Krishna S. Clinicomycological study of dermatophytes. Int J Pharm Biomed Res 2013; 4 (2): 132-134.

18. Rosenthal SA, Furnari D. Efficacy of "Dermatophyte Test Medium" Comparison of Two Commercial Preparations with Laboratory-Prepared Sabouraud-Antibiotic Medium. JAMA 1971; 104(5): 486-489.

\section{How to cite this article:}

Tokbipi Phudang, R., P. Baradkar Vasant and Shastri Jayanthi, S. 2019. Clinico-Mycological Study of Dermatophytosis and Dermatomycosis in Tertiary Care Hospital. Int.J.Curr.Microbiol.App.Sci. 8(01): 1297-1306. doi: https://doi.org/10.20546/ijcmas.2019.801.138 